\title{
Metodologia de Geração de Plataforma de Inteligência de Negócios para Comissões de Avaliação de Universidade
}

$$
\begin{aligned}
& \text { Alternative Title: Methodology for Generation of Business } \\
& \text { Intelligence Platforms to University Evaluation Committees }
\end{aligned}
$$

\author{
Caio Eduardo Ribeiro \\ Pontifícia Universidade Católica de Minas Gerais \\ Belo Horizonte, Brasil \\ 31980-110 \\ caioedurib@gmail.com
}

\section{RESUMO}

As técnicas de Inteligência de Negócios visam extrair conhecimento estratégico dos grandes volumes de dados das organizações. Com a necessidade de se gerenciar e manusear em tempo hábil esses dados faz-se necessária uma plataforma de Inteligência de Negócios. Este artigo apresenta uma metodologia de geração de plataformas para automação do processo organizacional de Comissões de Avaliação de universidade, que automatizam parte do processo e auxiliam na geração de conhecimento estratégico. A metodologia proposta abrange desde a especificação até a avaliação do protótipo da plataforma, feita segundo a norma ISO/IEC 9126. Foi criado um estudo de caso em uma Comissão de Avaliação de uma universidade, e plataforma desenvolvida obteve uma média geral de $88,9 \%$ de aprovação em sua avaliação, sendo considerada apta para implantação.

\section{Palavras-chave}

Inteligência de Negócios, Automação, Projeto de Software

\begin{abstract}
Business Intelligence techniques aim to extract strategic knowledge from great data volumes in organizations. With the need do manage and handle timely this data, a Business Intelligence Platform becomes necessary. This paper presents a methodology for generation of platforms for automation of organizational process from University Evaluation Committees, which automate part of the process and support strategic knowledge generation. The proposed methodology comprehends from specification to evaluation of the platform prototype, made according to ISO/IEC 9126 standard. A case study in an University Evaluation Committee was made, and the developed platform had an $88.9 \%$ approval rate, considered ready for deployment.
\end{abstract}

\section{Categories and Subject Descriptors}

H.3.5 [Online Information Services]: Web-based services

Permission to make digital or hard copies of all or part of this work for personal or classroom use is granted without fee provided that copies are not made or distributed for profit or commercial advantage and that copies bear this notice and the full citation on the first page. To copy otherwise, or republish, to post on servers or to redistribute to lists, requires prior specific permission and/or a fee.

SBSI 2015, May 26-29, 2015, Goiânia, Goiás, Brazil.

Copyright SBC 2015.

\author{
Anna Izabel J. Tostes Ribeiro \\ Pontifícia Universidade Católica de Minas Gerais \\ Belo Horizonte, Brasil \\ 31980-110 \\ annatostes@gmail.com
}

J.1 [Administrative Data Processing]: Education

General Terms

Measurement, Design, Standardization.

\section{Keywords}

Business Intelligence, Automation, Software Project

\section{INTRODUÇÃO}

As plataformas de Inteligência de Negócios (Business Intelligence - BI) combinam coleta e armazenamento de dados, gerenciamento de conhecimento e ferramentas de análise para apresentar informações internas e competitivas aos tomadores de decisão [11]. O grande volume de dados gerados pelas organizações torna difícil seu armazenamento e manuseio. Está implícita nessa definição a ideia de que plataformas de BI devem prover essas informações de forma segura e em tempo hábil, enquanto ainda sāo válidas para auxiliar na tomada de decisão [7].

A Comissão Própria de Avaliação (CPA) de uma universidade, tornada obrigatória pela Lei [15], é responsável por fazer todas as avaliações no âmbito da universidade, e encaminhálas ao Ministério da Educação, com o objetivo de manter a qualidade das instituições de ensino superior. As informações geradas pela análise dos resultados das avaliações aplicadas pela CPA devem ser entregues em tempo hábil para que possam ser úteis no auxílio à tomada de decisões administrativas da instituição. A falta de automação de alguns processos internos que demandam muito tempo dos membros da Comissão pode atrasar a entrega dos resultados.

Este artigo objetiva detalhar a metodologia de geração, e avaliação do protótipo de um sistema de BI desenvolvido na plataforma Sharepoint, com o objetivo de gerenciar e integrar os processos da Avaliação Institucional feita pela CPA da Pontifícia Universidade Católica de Minas Gerais (PUC Minas). Os resultados esperados são a automatização de parte do processo das avaliações realizadas, e a governança dos dados eletrônicos da organização. A plataforma desenvolvida foi avaliada segundo a norma ISO/IEC 9126 de avaliação de qualidade de software, e considerada apta para implantação.

Este artigo é apresentado em sete seções. Os conceitos de Inteligência de Negócios necessários para a compreensão do trabalho estão na seção 2 . A seção 3 indica trabalhos relacionados a este. A seção 4 contém uma descrição da metodologia de geração de software proposta, e a seção 5 apresenta um 
detalhamento do estudo de caso realizado para a CPA da PUC Minas. Os resultados da avaliação do sistema se encontram na seção 6. Finalmente, a seção 7 contém as conclusões e direções futuras para o trabalho.

\section{INTELIGÊNCIA DE NEGÓCIOS}

Inteligência de Negócios é uma evolução natural de uma série de sistemas anteriores, criados para dar suporte à tomada de decisões [7]. Esses sistemas podem ser divididos em seis categorias que funcionam de forma cíclica. Ou seja, os sistemas de uma categoria fornecem as entradas para os da categoria seguinte, até que uma nova iteração se inicie, conforme ilustrado na Figura 1.

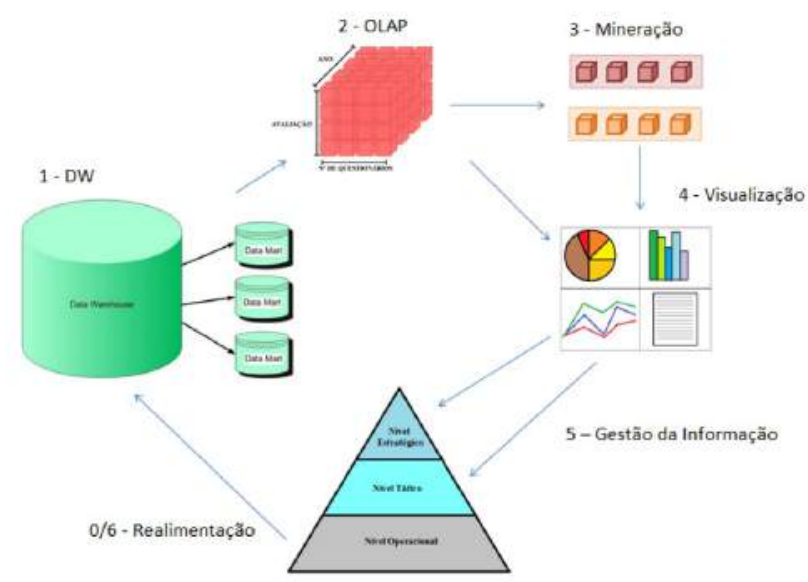

Figura 1. O Ciclo da Inteligência de Negócios

Um Data Warehouse (DW) é um grande Banco de Dados que contém os dados referentes às operações diárias da organização. Os DW são direcionados principalmente para aplicações de apoio à decisão, sendo otimizados para a recuperação de dados, e não para o processamento de transações como os Bancos de Dados (BDs) tradicionais [4]. Os DW possuem foco de auxílio à decisão, e servem às aplicações $O n$ Line Analytical Processing (OLAP), que otimizam a recuperação de dados multidimensionais para auxiliar análises estatísticas de diversas visões da organização.

Mineração de Dados (Data Mining) é o processo de identificar e interpretar padrões nos dados para se resolver um problema específico na organização. As ferramentas de Mineração utilizam técnicas de inteligência artificial e estatística avançada para descobrir fatos sobre os dados de forma automatizada [11]. As etapas do processo de Knowledge Discovery in Databses (KDD) proposto por [13] são Seleção, Pré-processamento, Transformação, Mineração de Dados e Avaliação, ou seja, a realização de mineração é necessária para gerar conhecimento através de uma Base de Dados.

Visualização é o nome do processo de representação de dados através de imagens gráficas. Tecnologias de visualização dão uma visão global de dados complexos, explorando a capacidade de sintetização da visão humana para criar imagens que representem as informações que devem ser transmitidas [7].

Estando as informações devidamente representadas, cabe às tecnologias de Gestão da Informação identificar o conhecimento que elas geram, e distribuí-lo adequadamente [10].
Os gráficos, painéis e relatórios dinâmicos que o processo realizado gerou vão chegar às pessoas que tomarão decisổes, auxiliando nas mesmas. As decisões são tomadas nos níveis Tático e Estratégico da organização, e propagadas para os níveis inferiores, podendo modificar os processos do nível Operacional. Novos dados são gerados pelos processos de rotina da organização, e estes são carregados no DW, reiniciando o ciclo.

\section{TRABALHOS RELACIONADOS}

Esta seção apresenta os principais trabalhos da literatura relacionados a este trabalho, $\mathrm{O}$ grupo Gartner, criador do termo Business Intelligence, avalia as plataformas de BI no artigo [8] e as classifica em categorias de acordo com a completude da visão e a habilidade de execução da plataforma. $\mathrm{Na}$ avaliação de 2013 a ferramenta Microsoft Sharepoint foi considerada a plataforma com maior habilidade de execução, e posicionada na categoria das plataformas líderes, pois sua completude de visão também é uma das melhores.

A arquitetura de um sistema de BI baseado em serviços web é apresentada em [9]. Os autores defendem que, no cenário empresarial atual, a obtenção de informações de suporte a decisão precisa ser ágil, e os sistemas precisam responder rapidamente às constantes mudanças dos requisitos do negócio e do ambiente. Um sistema baseado em serviços web possui essas e outras características desejadas, como a capacidade de coordenar a operação de várias plataformas ao mesmo tempo, boa escalabilidade, facilidade de implantação e adaptabilidade adequada para atender as mudanças no negócio. A arquitetura proposta no artigo é baseada na arquitetura de sistemas tradicionais de BI, e dividida em três camadas: uma camada Expressional, que contém a interface do sistema com os usuários; a camada Funcional onde os dados são tratados por funções de BI para serem apresentados; e a camada de Dados onde os servidores, estão localizados.

O artigo [6] descreve o desenvolvimento de uma solução de BI centrada em um DW, que utiliza integração de dados e OLAP para permitir, através de relatórios e gráficos, a visualização de dados financeiros e estratégicos de uma empresa em tempo real, dando apoio ao processo de tomada de decisão. Os autores descrevem as etapas do desenvolvimento do software e apontam os fatores principais para que um projeto de BI seja bem sucedido: definição clara dos objetivos, envolvimento das partes interessadas no projeto, estabelecimento de cronogramas e orçamentos e administração de expectativas. A plataforma de arquitetura escolhida para desenvolver o sistema foi o Microsoft Sharepoint, pois os relatórios são acessados via web, o que permite o acesso à informação em tempo hábil. Os autores constataram que o uso da interface web possui algumas limitações, como a manipulação limitada das dimensões de dados (os relatórios disponíveis são pré-formatados), e isso tornou necessário o uso de ferramentas especificas para a realização de algumas análises.

O estudo destes e outros trabalhos contribuiu para a formação da estratégia adotada na metodologia proposto, uma ferramenta web de automação de parte do processo operacional de uma CPA da PUC Minas, implantada na plataforma Sharepoint 2010. O objetivo do sistema é a otimização do processo interno e a realização da governança dos dados eletrônicos da organização. A metodologia desenvolvida tem foco nas possibilidades de um 
sistema web, principalmente na modularidade que a disponibilidade das informações permite.

\section{METODOLOGIA}

Esta seção detalha a metodologia de geração de software criada com o intuito de guiar o projeto, implantação e avaliação de sistemas de BI para Comissões de Avaliação de universidade. As ferramentas web precisam ter equilíbrio entre dinamismo e robustez, aptas a lidar com as constantes mudanças no negócio e otimizar o processo operacional da organização.

Para se aproveitar da flexibilidade e paralelismo possibilitados por uma plataforma web, é necessário que o processo não seja "engessado", e algumas de suas etapas possam ser realizadas ao mesmo tempo por pessoas ou equipes diferentes. No caso de uma avaliação de universidade, existem muitos aspectos a serem observados, e se eles forem examinados um a um, a avaliação se torna lenta e pesarosa. Por isso, este modelo propõe o conceito de Dimensões.

As Dimensões de uma avaliação são os aspectos diferentes da universidade que precisam ser verificados para garantir a qualidade do ambiente de ensino. O Ministério da Educação criou uma série de indicadores de qualidade que devem guiar a concepção das avaliações realizadas pela CPA, e através destes, é possível dividir a avaliação em uma série de Dimensões que podem ser consideradas individualmente. Isso permite que, por exemplo, uma equipe trabalhe na avaliação da dimensão Infraestrutura da Universidade ao mesmo tempo que outra trabalhe na avaliação da dimensão Pesquisa e Extensão.

A principal contribuição desta metodologia é o processo ser modularizado de forma a permitir que grande parte da descoberta de conhecimento seja realizada em paralelo, ou seja, partes do objetivo principal do processo serem produzidos ao mesmo tempo por equipes diferentes. Como a plataforma web integra o processo operacional da organização em um único sistema online, as informações estão disponíveis assim que são produzidas, e os fragmentos do produto final podem ser produzidos simultaneamente para posteriormente serem integrados, graças ao conceito de Dimensões. Além disso, a plataforma embarca as funções do KDD, automatizando-as na medida do possível, podendo no melhor caso fazer todas as operações necessárias on-line.

A Figura 2 mostra como o processo de KDD se torna mais dinâmico quando embarcado em uma plataforma de BI. As etapas de seleção, pré-processamento, transformação e mineração dos dados são realizadas pelas funções internas da plataforma, e as técnicas de visualização de BI facilitam sua interpretação para geração de conhecimento.

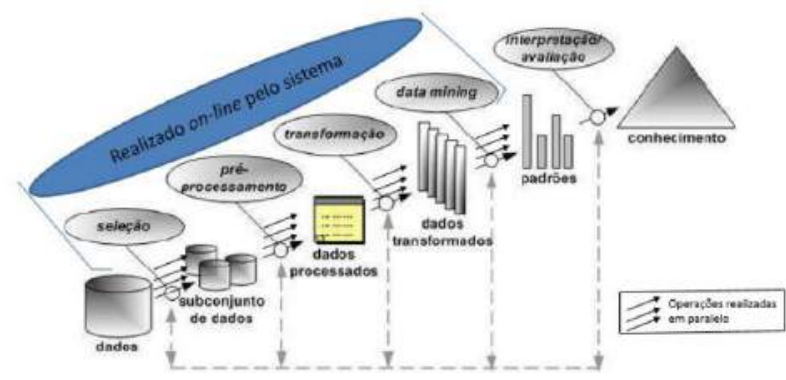

Figura 2. KDD na plataforma web de BI (adaptada de [13])
A metodologia criada é uma adaptação das diretrizes clássicas de projeto e desenvolvimento de software, com foco nos objetivos de sistemas web de automação e BI. Ela foi dividida em cinco fases interligadas, que se sobrepõem em partes da execução da metodologia, e sete diretrizes de projeto que se entrelaçam com essas fases.

\subsection{Fases da Metodologia}

A primeira fase é a de definição do Processo. Pode ser necessário um mapeamento do processo interno da organização, caso não haja documentação do mesmo, para determinar quais seus pontos críticos e como o software irá abordá-los. Se a organização já tiver um processo maduro e bem definido, a plataforma tem um desenvolvimento mais simples, mas este não é um caso comum especialmente no cenário brasileiro.

É de responsabilidade da equipe de desenvolvimento coletar informações sobre o processo operacional da organização, para traçar estratégias de como melhorá-lo com a plataforma de BI, e essa definição prévia é importante para que o produto gerado seja o que o cliente espera. Durante essa etapa, as avaliações devem ser dividas em Dimensões de acordo com os Indicadores do MEC abordados por elas.

Em seguida, é feito o mapeamento dos Dados. Nessa etapa é definido como os dados do sistema serão adquiridos e transformados. Os softwares de organizações frequentemente são responsáveis por prover acesso a dados vindos de diversas fontes, e é importante que haja um controle desse acesso, que também é definido nesta etapa. É importante ressaltar que, em um sistema de $\mathrm{BI}$, é comum que os dados sejam gerados com alta frequência e grande volume, e o sistema deve estar preparado para lidar com isso.

A terceira fase é a do Projeto do software, que deve determinar exatamente o seu escopo e objetivos. O projeto é usado como guia durante as etapas seguintes para garantir que a equipe de desenvolvimento e os clientes estejam alinhados quanto ao produto final do projeto. Cada solução do sistema deve ser projetada individualmente, com seus dados de entrada e saída, e as operações necessárias para alcançar seus objetivos. Com o projeto modular, é possível o desenvolvimento paralelo das soluções do sistema pela equipe. Esta etapa também envolve a definição de como o sistema irá se apresentar para o usuário: seu layout (preferivelmente com ícones e botões, e no formato de uma página web como as que os usuários estão habituados). Finalmente, deve ser determinado de que maneira o usuário será treinado a utilizar o sistema e as formas de ajuda que estarão disponíveis.

Com a finalização do projeto, entra a fase de Desenvolvimento. Todos os sites da plataforma web são desenvolvidos, e as soluções nativas do Sharepoint e as customizadas para o sistema são implementadas de acordo com o projeto realizado. Entregas parciais são recomendadas, porque elas permitem que o projeto amadureça antes do produto final ser entregue. O principal objetivo de um sistema de BI é a geração de conhecimento estratégico em tempo hábil, e essa deve ser a principal preocupação da equipe de desenvolvimento durante todo o processo, especialmente na otimização de suas soluções.

Neste tipo de projeto, é comum que hajam alterações nos requisitos do sistema durante a etapa de desenvolvimento, por 
mais que estas sejam evitadas nas etapas anteriores. A equipe de desenvolvimento deve estar preparada para fazer controle de alterações e definir como elas vão impactar no projeto como um todo.

Finalizando o processo, temos a etapa de Avaliação. Para determinar se o sistema atingiu os objetivos definidos em seu projeto, é feita uma avaliação quantitativa que reflete a qualidade do software em números que podem ser comparados com avaliações de versões futuras, e guiar a equipe de desenvolvimento nos próximos projetos para o sistema. A avaliação é feita seguindo a norma ISO/IEC 9126, e pode ser aplicada em forma de questionário ou entrevista, dependendo do projeto. As cinco etapas da metodologia são ilustradas pela Figura 3.

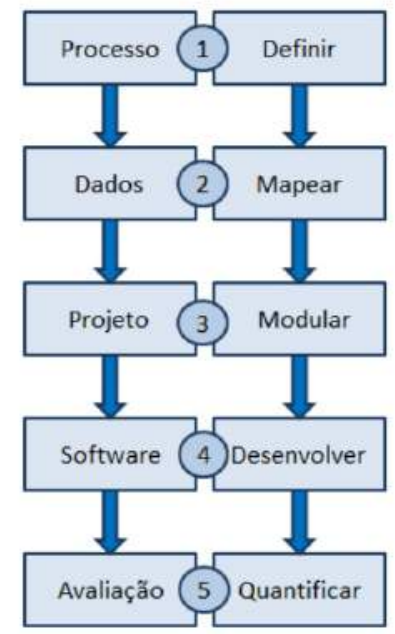

Figura 3. Etapas da metodologia de geração software

\subsection{Diretrizes para o projeto da plataforma}

Antes de se iniciar o desenvolvimento de uma plataforma de BI, é muito importante que ela seja projetada adequadamente. Os stakeholders (pessoas envolvidas com o projeto, principalmente os clientes) e os desenvolvedores precisam entrar em acordo prévio sobre todos os detalhes do produto a ser entregue. Para o desenvolvimento de uma estratégia para o projeto de uma plataforma feita em Microsoft Sharepoint, são apresentados em [3], sete diretrizes a partir das quais foi definida estratégia guia para as cinco fases da metodologia:

1. Propósito (Purpose): Identificação dos objetivos da plataforma, que precisam estar bem definidos antes do início da implementação. Abordado na primeira e na terceira fases.

2. Controle (Governance): Compreende uma documentação dos processos, padrões, papéis, responsabilidades e as políticas que são usadas na organização. Em suma, todas as regras e características do negócio. Abordado na primeira e segunda fases.

3. Pessoas e Objetivos (People and Objectives): Identificação das audiências que possuem participação no ambiente da plataforma. É importante que se compreenda quem são essas pessoas, e quais são as tarefas que elas precisam desempenhar. Abordada na primeira fase.

4. Requisitos e Análise (Requirements and Analysis): Através de entrevistas com os stakeholders do sistema, reuniões das equipes de desenvolvimento com os clientes, entrevistas com usuários finais, entre outros, os requisitos do sistema são formalmente coletados e documentados. Abordada na terceira fase.

5. Arquitetura da Informação (Information Architecture): Define as abordagens de duas áreas-chave: Organização da Informação e Acesso à Informação. Importante para a realização da governança dos dados da organização. Abordada nas segunda e terceira fases.

6. Tecnologia (Technology): As considerações de tecnologia incluem instalação, configuração e manutenção de ambos o hardware e o software necessários para o uso da plataforma, bem como a integração do ambiente Sharepoint com os sistemas da organização. Abordada na terceira e na quarta fases.

7. Manutenção e Melhoria (Maintenance and Enhancement): Compreende métricas de avaliação de desempenho da aplicação, que avaliam tanto a satisfação do usuário final quanto o benchmark da plataforma. Para lidar com as mudanças que se mostrarem necessárias, enquanto o negócio se modifica e evolui, a plataforma precisa ter suas funcionalidades constantemente avaliadas, permitindo que os desenvolvedores tomem decisões relativas às mudanças da aplicação. Abordada na última fase.

O planejamento da plataforma deve ser uma tarefa minuciosa, para que todos os detalhes do processo estejam bem definidos previamente. A Engenharia de Software recomenda não se poupar tempo e recursos nessa etapa, porque gerenciar mudanças de projeto durante seu desenvolvimento pode prejudicar bastante o cronograma e até inviabilizar o projeto. Isso é ainda mais crítico em uma plataforma que abarca tantas partes do processo de uma organização, desde a gestão dos seus dados e pessoas até a geração de produtos e conhecimento. Deve-se atentar para as áreas mais críticas e que possuem necessidades mais abrangentes de informações gerenciais [Barbieri 2001].

As primeiras reuniões entre a equipe de desenvolvimento e os stakeholders são para definir o Propósito da plataforma, ou seja, seus objetivos perante a organização e suas atividades. Definidos os resultados esperados do projeto, as reuniões seguintes devem explicitar as características de Controle e as Pessoas e Objetivos relevantes. É recomendado que sejam criados grupos de usuários, para garantir a seus membros o acesso a funcionalidades do sistema, e cada usuário deve ser alocado em um ou mais grupos, de acordo com as tarefas que deve desempenhar.

A diretriz seguinte é a de Requisitos e Análise na qual, através de entrevistas com os stakeholders do sistema, reuniões das equipes de desenvolvimento com os clientes, entrevistas com usuários finais, entre outros, os requisitos dos softwares da plataforma são formalmente coletados e documentados. Os requisitos devem ser classificados em três categorias, de acordo com sua relevância. São considerados Essenciais os requisitos imprescindíveis para que o software funcione, Importantes aqueles sem os quais o software funciona, mas de forma não 
satisfatória, e Desejáveis os que não comprometem as funcionalidades básicas do software, e podem ser deixados para versões futuras do sistema caso não haja tempo hábil para implementá-los na versão que está sendo especificada.

Para a descrição da Arquitetura da Informação, são definidos os dados que a plataforma precisará gerenciar, e como será sua organização. Antes da implementação iniciar, a equipe deve ter uma ideia clara de como irá obter e administrar esses dados, e escolher uma arquitetura que aplique corretamente as regras do negócio, além de definir as ferramentas de gestão de dados que serão utilizadas.

Nesta metodologia, foi definido que seria utilizado o padrão de projeto MVC (padrão Model-View-Controller, amplamente adaptado para uso em aplicações Web [14]) para o desenvolvimento das Web Parts Customizadas, softwares desenvolvidos especialmente para cumprir uma função do processo, que são embarcados na plataforma pelo Sharepoint. Durante a descrição da Arquitetura da Informação, também são especificadas as ferramentas a serem utilizadas e a forma de comunicação entre a plataforma e suas fontes de dados. Essa parte da diretriz e a diretriz de Tecnologia são realizadas em conjunto, pois a equipe de Tecnologia da Informação da universidade é responsável por considerar as necessidades de hardware e software que as decisões de arquitetura implicam.

\section{Estudo de caso}

Para testar a metodologia proposta, foi feito um estudo de caso com o desenvolvimento de uma plataforma de BI para a CPA da PUC Minas. A etapa de avaliação foi descrita durante a apresentação dos resultados da avaliação dessa plataforma, na seção seguinte. Não serão apresentadas todas as etapas do processo, pois para o escopo deste trabalho é necessário abordar apenas as essenciais para a compreensão do sistema desenvolvido e como a metodologia auxiliou em seu projeto, implantação e avaliação.

\subsection{Melhoria no processo de avaliação}

A plataforma desenvolvida é um protótipo que inicialmente tem como foco automatizar os processos operacionais realizados na CPA da PUC Minas, porém foi planejada de maneira escalável, permitindo que em trabalhos futuros sejam acrescentadas novas funcionalidades.

Foi definido que o sistema deveria automatizar a Avaliação Institucional realizada pela organização, pois esse é o processo mais custoso realizado na CPA da PUC Minas. A Figura 4 mostra as principais etapas do processo de avaliação abordado, antes de ser modificado para o sistema. As cores diferentes indicam sistemas dos quais o processo dependia para seguir adiante, $o$ que muitas vezes causava atrasos devido à mão de obra especializada necessária, e necessidade de colaboração de equipes envolvidas em outros projetos. Grande parte do processo era realizado através do trabalho manual dos funcionários da $\mathrm{CPA}, \mathrm{e}$ não havia nenhum sistema que gerenciasse os documentos eletrônicos da organização, causando problemas de versionamento e disponibilidade dos dados.

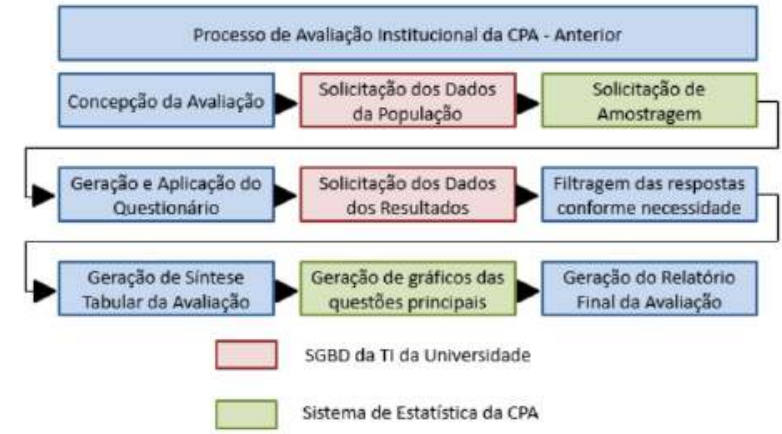

Figura 4. Processo de Avaliação anterior

Nessa etapa, foram identificadas as fontes de dados e como deveria ser a comunicação entre a plataforma e os demais sistemas para que a plataforma mantivesse o controle sobre o processo. Uma decisão importante foi aumentar a granularidade dos relatórios da avaliação, o principal produto do processo, proposta trazida por este trabalho. Com o objetivo de permitir que partes do relatório fossem geradas em paralelo por usuários diferentes, foram definidas as Dimensões da avaliação em questão, e esse conceito foi usado na implementação das soluções desenvolvidas.

O processo modificado para a Avaliação Institucional é exibido na Figura 5. Foram desenvolvidas Web Parts na plataforma para gerar automaticamente a amostra, os gráficos, as Sínteses Tabulares e o Relatório Final (reunindo dados das Dimensões, para edição dos especialistas da (PA), e todos os dados necessários para a realização das operações da organização foram negociados com seus responsáveis, e centralizados em uma Base de Dados local, sob gestão da TI da universidade. Dessa forma, o processo passou a não depender de outros sistemas. Além disso, a plataforma deveria fazer a gestão dos documentos eletrônicos da organização, incluindo o controle de acesso aos mesmos e seu versionamento. O processo modificado é reduzido e automatizado em grande parte, tornando a operação da organização mais ágil e eficiente, e permitindo mais atenção à qualidade do produto.

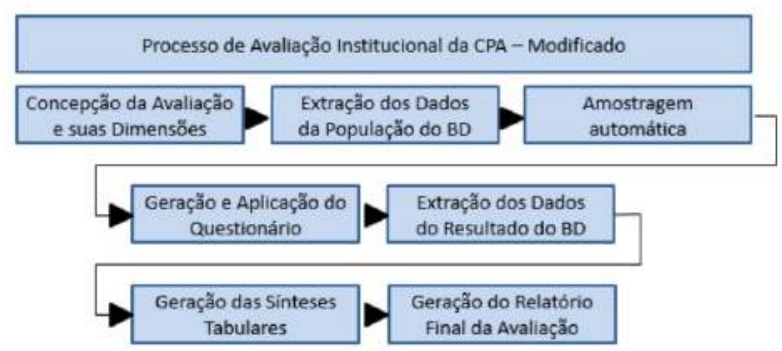

Figura 5. Processo de Avaliação Modificado

\subsection{Plataforma desenvolvida}

A navegação do SIAV se baseia principalmente no menu de links encontrado à esquerda da tela de todas as páginas, chamado Quick Launch, que é sensível ao contexto, e exibe os links mais relevantes para o usuário de acordo com onde ele se encontra no sistema. O conteúdo das páginas da aplicação é facilmente 
alterável pelos usuários com permissão de edição de página, podendo ser adaptado às mudanças dos processos sem a necessidade do desenvolvimento de uma nova versão. Isso torna a ferramenta adaptável e flexível, necessidade inerente a sistemas com grande teor de modificações, e diminui a necessidade de manutenções custosas que interrompem o processo operacional.

Todas as soluções nativas utilizadas no SIAV se baseiam nas funcionalidades de Listas do Sharepoint, definidas como uma coleção de informações compartilhadas entre membros de uma equipe. As Listas predefinidas do Sharepoint incluem listas de eventos, calendários, quadros de discussão, além da possibilidade de conectar as listas a fontes de dados externas e de criar listas customizadas.

Um tipo especial de lista é a Biblioteca de Documentos, otimizada para armazenar documentos, planilhas, slides ou outros tipos de arquivos, e foi esse tipo de lista o escolhido para facilitar a gestão eletrônica dos documentos da CPA. Usando essa lista, os usuários têm garantia de que todos estão trabalhando com os mesmos arquivos, podem ver as alterações realizadas nos mesmos, e ter a segurança de apenas uma edição estar sendo feita naquele momento (funcionalidade de check-in e check-out). Através dessas listas, o sistema realiza a governança de dados da organização, centralizando-os em um só servidor e permitindo seu versionamento e controle de alteração.

Existem partes do processo da avaliação que não podem ser realizadas pelas soluções nativas do Sharepoint. Para essas partes, foram desenvolvidas soluções customizadas, programas com funções que vão tornar o processo da CPA mais rápido e eficiente, com alto grau de confiabilidade, visto que os programas são menos suscetíveis a erros do que o trabalho humano.

As Web Parts customizadas foram desenvolvidas nas linguagens ASP.NET e C\#, na IDE Visual Studio 2010, que é a recomendada pela Microsoft para a construção de soluções de Sharepoint 2010 [12]. Para realizar as consultas ao BD necessárias, foi utilizada a biblioteca gratuita SqlConnection, que cria uma sessão de conexão com o Banco para aquela aplicação, executa queries e retorna seus resultados dentro do contexto da conexão estabelecida. Para a geração dos documentos do Word 2010 e planilhas do Excel 2010 criados pelas Web Parts, foi utilizada a biblioteca gratuita Open XML SDK, que permite a escrita de um arquivo XML (eXtensible Markup Language, o formato padrão dos arquivos do Microsoft Office 2010) sem a necessidade de utilizar os programas do Office para criar os arquivos. Finalmente, para a geração de imagens JPEG (gráficos gerados automaticamente) foi utilizada a biblioteca gratuita Interop.Excel, da Microsoft, que inicia uma instância do Excel na máquina do servidor, e parametriza a criação e exportação da imagem do gráfico.

Na CPA da PUC Minas, uma das primeiras etapas do processo de avaliação por questionário é a seleção do conjunto de respondentes, ou seja, dos indivíduos para os quais o questionário será aplicado. A esse conjunto é dado o nome de amostra, e sua seleção é sempre aleatória dentre um grupo predefinido de indivíduos, chamado conjunto universo. A Web Part de Geração de Amostras permite que o usuário selecione um ou mais conjuntos-universo (conjunto de registros a ser considerado o total para aquela categoria), e o número de respondentes desejado para cada amostra a ser gerada, e faz a seleção aleatória para o usuário, criando uma planilha Excel com os dados dos registros selecionados.
Para facilitar a visualização dos dados obtidos pela aplicação de questionários, os relatórios da CPA apresentam esses dados na forma de gráficos, que são analisados pelos especialistas da Comissão, compondo o relatório de avaliação. A geração desses gráficos é realizada por uma Web Part que permite ao usuário selecionar uma ou um grupo de questões de algum questionário aplicado, e em seguida informe as configurações desejadas para o gráfico a ser gerado. Para cada gráfico é adicionada automaticamente uma imagem na Lista de Imagens, com uma numeração correspondente à Dimensão do relatório a qual ela pertence informada no nome da imagem. Além da imagem, também é gerada uma planilha-fonte com os dados que foram usados na construção do gráfico, para conferência, e esta é adicionada a uma lista de planilhas-fonte, na mesma página.

Além do relatório de avaliação, a CPA gera documentos de Síntese Tabular para facilitar a análise dos resultados obtidos pelos interessados. O documento de Síntese possui uma tabela para cada questão selecionada, apresentando os resultados obtidos para aquela questão de forma sucinta. Para gerar a Síntese, o usuário deve selecionar o conjunto de questões e escolher o nome do arquivo, e uma Web Part customizada criada para gerar esses documentos cria e adiciona a Síntese à lista destas, presente na mesma página.

O produto principal da Avaliação Institucional é seu relatório final, que contém as análises da CPA acerca dos resultados obtidos na aplicação do questionário. Esse relatório é formado por várias partes, os chamados Documentos de Dimensão, que ficam armazenados na Biblioteca da página de Dimensões do site. Cada item dessa lista é um documento que será integrado no relatório final. Além dos documentos de Dimensão, o relatório também contém os gráficos e análises dos resultados das questões dos questionários aplicados naquela avaliação. Para facilitar o trabalho de construção do relatório, uma Web Part de geração de relatório acessa as listas de Documentos de Dimensão e Imagens do site, e monta um relatório parcial, reunindo esses fragmentos.

Em resumo, o SIAV realiza a governança dos dados da organização, permite a colaboração on-line dos usuários, realiza o controle de acesso às funcionalidades automaticamente, e automatiza partes importantes do processo. Como é um sistema web, a flexibilidade e possibilidade de expansão do SIAV são adequadas para o cenário de mudanças constantes de uma organização cujo processo está em constante evolução.

\section{AVALIAÇÃO QUANTITIVA DA PLATAFORMA}

Uma avaliação quantitativa deve permitir uma noção exata do quanto o software cumpre as expectativas dos clientes. O SIAV foi avaliado de acordo com a norma ISO/IEC 9126 [1], criada com o intuito de servir como uma padronização da avaliação da qualidade de produtos de software, que estabelece uma avaliação quantitativa que pode ser repetida a cada nova versão da plataforma.

A norma ISO/IEC 9126 tem foco na qualidade do produto de software, propondo Atributos de Qualidade, distribuídos em seis características principais, cada uma delas divididas em sub-características que serão avaliadas, exibidas na Figura 6. O método escolhido para a avaliação do SIAV foi a 
entrevista, devido ao número reduzido de usuários (dez funcionários da organização, que iriam utilizar o sistema).

\begin{tabular}{|c|c|c|c|}
\hline \multirow{6}{*}{$\stackrel{v}{\sim}$} & \multirow[b]{2}{*}{ Funcionalidade } & Adequação & Sequrance \\
\hline & & Acurácia & Conformidade \\
\hline & \multirow{2}{*}{ Confiabilidade } & Maturidade & Recuperabilidade \\
\hline & & Tolerância a Falhas & Conformidade \\
\hline & \multirow{3}{*}{ Usabilidade } & Inteligibilidade & \\
\hline & & Apreensibilidade & $\begin{array}{l}\text { Atratividade } \\
\text { Conformidade }\end{array}$ \\
\hline & & Operabilidade & \\
\hline \multirow{7}{*}{ 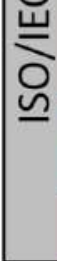 } & \multirow[b]{2}{*}{ Portabilidade } & Adaptabilidade & Substituição \\
\hline & & Facilidade de Instalação & Conformidade \\
\hline & \multirow{3}{*}{ Manutenibilidade } & Analisabilidade & \\
\hline & & Modificabilidade & Testabilidade \\
\hline & & Estabilidade & Conformidade \\
\hline & \multirow{2}{*}{ Eficiência } & Consumo de Recursos & Conformidade \\
\hline & & Consumo de Tempo & \\
\hline
\end{tabular}

Figura 6. Características da ISO/IEC 9126

Finalizando as etapas da metodologia de geração de software proposta, foram feitas entrevistas com os funcionários da CPA nas quais cada entrevistado deveria dar uma nota para a plataforma avaliada, para cada sub-característica das características avaliadas na norma ISO/IEC 9126. O foco do projeto da plataforma foi na sua funcionalidade e usabilidade, características que determinam o índice de adesão da plataforma, porque o sistema está em sua primeira versão, e ainda não havia sido implantado. Para as características mais importantes, foi dado um peso maior, de forma que as médias obtidas nestas duas características valessem duas vezes mais no cálculo da média geral obtida pelo sistema. Se essas notas fossem decididas pelo avaliador, analisando as respostas dos usuários de perguntas sobre o sistema, os resultados poderiam se tornar tendenciosos, portanto a entrevista reflete melhor a opinião do usuário se a nota for decidida no momento da entrevista, em conjunto com ele [5] (naturalmente, o papel do entrevistador é apenas tirar dúvidas a respeito do conceito a ser avaliado, sem influenciar de maneira alguma na resposta do entrevistado).

Foram entrevistados 10 funcionários da CPA, e a média aritmética simples das notas de cada entrevistado foi considerada a nota da plataforma para a sub-característica em questão. A média aritmética simples das notas das sub-características foi considerada a nota final da plataforma para característica, e a média geral é uma média ponderada das notas de todas as características, considerando um peso de dois para as características de Funcionalidade e Usabilidade.

Antes da realização das entrevistas, a plataforma foi apresentada para o entrevistado, e suas e funcionalidades foram explicadas detalhadamente, além dos objetivos estabelecidos no projeto. Em seguida, foi explicado ao entrevistado cada subcaracterística a ser avaliada, e ele poderia escolher uma nota entre 0 e 10 , ou se abster de dar sua opinião para aquela subcaracterística, caso não achasse que haviam informações suficientes para formá-la. Não houveram limitações de tempo para a resposta das perguntas. Os entrevistados tiraram suas dúvidas sobre a plataforma no momento da entrevista, e deram sugestões de melhorias que foram discutidas em reuniões com os stakeholders e, caso aprovadas, implementadas em seguida.
Foi estabelecido um objetivo de $80 \%$ de aprovação para que a plataforma fosse considerada totalmente aprovada. $\mathrm{O}$ gráfico da Figura 7 mostra os resultados obtidos pela plataforma na avaliação de cada característica, e a média geral final. O SIAV foi aprovado totalmente em sua avaliação geral, e a única característica que não atingiu a meta foi a de Confiabilidade. Isso ocorreu porque a sub-característica de Tolerância a Falhas, que avalia a reação da plataforma em situações adversas, recebeu notas baixas, pois ainda não haviam sido implementadas funções de comunicação eficientes, que informassem ao usuário que ações tomar em caso de falha. Aumentar a confiabilidade do sistema deve ser, portanto, um dos focos no projeto das próximas versões.

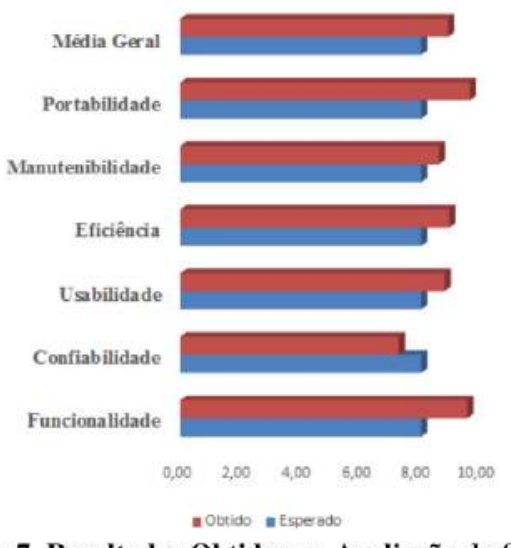

Figura 7. Resultados Obtidos na Avaliação do Sistema

\section{CONCLUSÕES}

Este trabalho apresentou uma metodologia para guiar o projeto e desenvolvimento de plataformas de BI que automatizam parte do processo operacional de Comissões de Avaliação de universidade. Foram descritos os detalhes de estruturação da plataforma e as tecnologias utilizadas, de forma que ela possa ser replicada no projeto de plataformas com características semelhantes. A plataforma desenvolvida para testar a metodologia foi avaliada segundo a norma ISO/IEC 9126, e obteve uma pontuação média de $88,9 \%$, sendo considerada aprovada.

O uso do Microsoft Sharepoint para desenvolvimento de plataformas web de colaboração e governança de dados é endossado porque, apesar do alto custo financeiro e da necessidade de técnicos treinados para administrar a aplicação, a ferramenta traz flexibilidade, disponibilidade e grande capacidade de execução para o projeto. As aplicações podem ser aprimoradas e passar por manutenção sem ter seu funcionamento interrompido, a maior parte do tempo. Os documentos da organização são centralizados, aumentando sua disponibilidade e diminuindo o custo de armazenamento e gestão, sem perder o controle de acesso e versionamento dos mesmos.

Durante a modelagem do processo operacional da organização, foram identificadas as funções críticas e como tornalas mais eficientes, e foi decidido que o processo da avaliação deveria ser dividido em Dimensões e indicadores, o que tornou o processo mais dinâmico e permitiu a realização paralela de algumas de suas etapas. A plataforma desenvolvida automatiza parte dos processos da organização, além de realizar a gestão eletrônica de seus documentos. A estrutura do SIAV é adaptável 
para qualquer avaliação baseada em questionário, o que torna o sistema expansível para os outros processos da CPA além dos abordados, e faz com que a metodologia apresentada possa ser adotada sem muitas alterações por outras organizações que utilizam essa forma de avaliação.

As direções futuras deste trabalho incluem a implantação de um Data Warehouse da CPA, para que as avaliações possam apresentar a progressão de vários aspectos da universidade ao longo do tempo. O sistema também será adaptado para incluir módulos para as outras avaliações realizadas pela organização, e um módulo de gestão de processos internos.

\section{REFERÊNCIAS}

[1] Abran, A. 2010. Software Metrics and Software Metrology. John Wiley \& Sons Inc.

[2] Barbieri, C. 2001. BI-Business Intelligence: modelagem e tecnologia. Axcel Books

[3] Carr, J. 2011. Case study: Developing a Sharepoint 2010 strategy. . or how setting it up and getting it out there is not a strategy. Bulletin of the American Society for Information Science and Technology, v. 37, p. 26-28.

[4] Chaudhuri, S.; Dayal, U. 1997. An overview of Data Warehousing and OLAP technology. SIGMOD Rec., ACM, New York, NY, USA, v. 26, n. 1, p. 65-74.

[5] Lakatos, E., Marconi, M. 2010. Fundamentos de metodologia científica. Atlas.

[6] Muszinski, A.; Bertagnolli, S. de C. 2009. Business Intelligence: um sistema de apoio a decisões gerenciais.

[7] Negash, S. 2004. Business intelligence. Communications of the Association for Information Systems, v. 13, p. 177 -195.
[8] Schlegel, K. et al.. 2013. Magic quadrant for business intelligence and analytics platforms

[9] Shi, C.; Zhang, D. 2010. Web service-based business intelligence system research and implementation. In: Innovative Computing Information and Control, 2008. p. 200-204.

[10] Turban, E. et al. 2010. Tecnologia da Informação para Gestão: Transformando os Negócios na Economia Digital. Bookman.

[11] Xu, L. et al. 2007. Research on business intelligence in enterprise computing environment. In: Systems, Man and Cybernetics, 2007. ISIC. IEEE International Conference.

[12] English, B., Alderman, B., and Ferraz, M. 2010. Microsoft SharePoint 2010 Administrator's Companion. Microsoft Press, $1^{\text {st }}$ Edition.

[13] Usama M. Fayyad, Gregory Piatetsky-Shapiro, and Padhraic Smyth. 1996. From data mining to knowledge discovery: an overview. In Advances in knowledge discovery and data mining, Usama M. Fayyad, Gregory Piatetsky-Shapiro, Padhraic Smyth, and Ramasamy Uthurusamy (Eds.). American Association for Artificial Intelligence, Menlo Park, CA, USA 1-34.

[14] Avraham Leff and James T. Rayfield. 2001. Web-Application Development Using the Model/View/Controller Design Pattern. In Proceedings of the 5th IEEE International Conference on Enterprise Distributed Object Computing (EDOC '01). IEEE Computer Society, Washington, DC, USA, 118-.

[15] BRASIL, 2004. Lei de $\mathrm{n}^{\circ}$ 10.861, artigo 11, 14 de Abril de 2004. Disponível em $<$ http://www.planalto.gov.br/ccivil_03/_ato20042006/2004/lei/l10.861.htm> acesso em 04/03/2015 